\title{
Magnetization reversal in magnetostatically coupled dot arrays
}

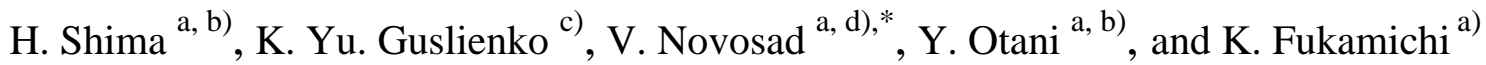 \\ a) Department of Materials Science, Graduate School of Engineering, Tohoku University, \\ Sendai 980-8579, Japan \\ b) JST, Japan Science \& Technology Corporation, CREST, Kawaguchi 3320012, Japan \\ c) School of Physics, Korea Institute for Advanced Study, Seoul 130-012, Korea \\ ${ }^{d)}$ Materials Science Division, Argonne National Laboratory, Argonne, IL 60439, USA
}

\begin{abstract}
The submicron permalloy dots with variable diameter and interdot distance were microfabricated into a rectangular lattice by means of e-beam lithography and lift-off techniques. The hysteresis loops exhibit characteristic magnetization reversal accompanied by "nucleation" and "annihilation" of magnetic vortices inside the dots. The magnetic response of the samples with well-separated elements is isotropic in the plane. The arrays with a small interdot distance show magnetic anisotropy with the easy axis along the shortest period in the array. This anisotropy is originated from the interdot magnetostatic interaction. The magnetostatic interaction is found to play an important role in determining the magnetization process for the dot arrays. For example, in the closely-packed (when interdot distance is smaller than dot radius) arrays so that $d / R<1$, the magnetostatic interaction decreases the vortex nucleation and annihilation fields, and increases the initial susceptibility.
\end{abstract}


The arrays of sub-micron size ferromagnetic particles draw much attention because of their possible potential as magnetic storage media [1] as well as a model system for studying magnetization reversal processes [2]. Small dots reveal a single-domain state to reduce exchange energy, but non-uniform magnetic states stabilize as the dot sizes are increased. For example, an isolated polycrystalline ferromagnetic disk-shaped dot with submicron diameters and thickness of several ten nanometers exhibits a vortex type spin structure with a closure flux in remanence [3-7]. The magnetic behavior of dot arrays is defined by the dot individual properties for well-separated magnetic elements, whereas the interdot magnetostatic interaction becomes essential for the dot arrays with small interdot distances [8-11]. In the present work we report the experimental results of magnetization reversal in submicron circular Permalloy ( $\left.\mathrm{Py}, \mathrm{Fe}_{81} \mathrm{Ni}_{19}\right)$ dots arranged into rectangular lattices, wherein the magnetization reversal accompanies nucleation, displacement and annihilation of magnetic vortices. We will show that the magnetostatic interaction plays an important role in determining the magnetization reversal for the arrays with a small interdot distance, leading to considerable decreases in the vortex nucleation $H_{n}$ and annihilation $H_{a n}$ fields, and an increase in initial in-plane susceptibility $\chi(0)$.

The arrays of circular Py dots were prepared using micro-fabrication techniques as follows. Firstly a standard silicon wafer is spin-coated with a positive photoresist. Next, the desired patterns are then defined by e-beam lithography. The magnetic film is deposited in vacuum on the watercooled substrate from a Py target. The deposition rate for all the prepared samples is about 0.1 $\mathrm{nm} / \mathrm{s}$. The as-deposited reference film is magnetically soft with a coercive field of several Oersteds. Finally, after ultrasonic assisted lift-off process, we obtained the arrays of circular dots arranged into rectangular lattices with variable diameter and interdot distance. The orientation of the lattice is shown in Fig. 1. The dot thickness $L$ is about $80 \mathrm{~nm}$ and diameters $2 R$ are chosen to be $0.4 \mu \mathrm{m}$, $0.6 \mu \mathrm{m}$, and $0.8 \mu \mathrm{m}$. For studying the effect of magnetostatic interdot interaction, the distance between edges of neighboring elements $d$ was varied from $30 \mathrm{~nm}$ to $1 \mu \mathrm{m}$. We prepared two kinds of the rectangular dot arravs: A) interdot distance $d_{\mathrm{x}}$ parallel to $O x$ axis is variable and decreases 
from $2 R$ to almost zero, whereas $d_{\mathrm{y}}=2 R$ is constant; B) $d_{\mathrm{x}}=R / 2$ is constant, $d_{\mathrm{y}}$ decreases from $3 R$ to $R / 2$ (square array), $\mathrm{R}=0.4 \mu \mathrm{m}$. The microfabricated circular dots have sharp edges and are almost identical, as confirmed by an ex-situ atomic force microscope (AFM), a scanning electron microscope (SEM) and a magnetic force microscope (MFM). The hysteresis loops were traced using a magneto-optical technique and a resonating sample magnetometer (RSM) for different orientations of the dot arrays with respect to the in-plane applied magnetic field. The domain structure was observed in remanence with a magnetic force microscope (MFM).

Figure 1 shows SEM images and corresponding hysteresis loops measured along the [01] lattice direction of the A-type dot arrays with diameter of $0.6 \mu \mathrm{m}$ and variable interdot distances. The magnetization reversal process is observed to accompany the "nucleation" and "annihilation" of magnetic vortices [6,7]. With decreasing field from the saturated state, the magnetization gradually decreases, showing an abrupt jump at the nucleation field $H_{\mathrm{n}}$. In this field a single magnetic vortex is formed in each dot. In the remanent state, the vortex stays at the center of the dot, as confirmed by MFM imaging. When the external magnetic field is applied, the vortex core is displaced to increase the average magnetization component along the field direction according to the balance between the magnetostatic and Zeeman energies. At the annihilation field $H_{\text {an }}$, the vortex vanishes and turns to a single-domain state. The dot-to-dot geometry and the crystal structure variations broaden the distribution of the vortex nucleation and annihilation fields. The microfabrication processes for all samples are identical. Therefore, the intrinsic distribution of $H_{n}$ and $H_{a n}$ has to be of the same order for both magnetostatically isolated and coupled dot arrays. This fact was confirmed by directly comparing the broadening of switching fields normalized by their average values for the samples with different diameters and interdot distances. This contrasts with the experimental data reported for rectangular Py particles, where the distribution in switching field was found to get narrower due to the magnetostatic interaction between the elements [11]. Note that structural or micro-structural defects have stronger influence to broadening of nucleation field 
than the annihilation field values. As seen from Fig. 1, the switching fields and the slope of the linear part of hysteresis loops depend on the interdot distance.

Figure 2 summarizes the experimental data for the magnetostatically induced anisotropy constant $K_{u}$ and the vortex annihilation field in the A-type arrays with the variable interdot distance $d_{x}$. The value of $d_{y}$ was kept constant and equal to the dot diameters. The magnetic field is applied along the [10] axis. The values of $H_{a n}$ and $H_{n}$ are normalized to their values measured in arrays of well-separated dots $\left(d_{x}, d_{y}>>\mathrm{R}\right)$, and the interdot distance is shown in reduced units $\delta=d / R$. The anisotropy constant was determined as $K_{u}=\Delta H_{a n} M_{s} / 2$, where $\Delta H_{a n}=H_{a n}($ isol $)$ - $H_{a n}$ is difference between the annihilation fields in arrays of isolated and coupled dots. $M_{s}=8.6 \times 10^{5} \mathrm{~J} / \mathrm{m}$ is the magnetization of saturation for permalloy. The magnetostatic interdot interaction is important in the magnetization reversal for arrays with $\delta=d / R<0.5$, leading to a decrease in both, nucleation and annihilation fields. Similar results were obtained with micromagnetic calculations for the chain of ferromagnetic disks [12].

The closure of the magnetic flux structure, that is realized in circular ferromagnetic dots with vortex spin distribution, suggests that dots are not interacting in remanence. The contribution of the out-of-plane magnetization component in the vortex core can be ignored, because the vortex core radius, being approximately equal to exchange length, is small in comparison to the in-plane dot size. However, field-driven displacement of the vortices results in a non-negligible stray field around the dot, which defines the strength of interdot magnetostatic coupling. The magnetic properties of identical interacting dots can be modeled analytically on the basis of a "rigid vortex" model [6]. This model assumes that the vortex moves in in-plane applied magnetic fields while keeping its shape. The reduced equilibrium core displacement $s=l / R$ is determined by minimizing the total magnetic energy as a function of the dot sizes and interdot distances. In external magnetic field, the dot energy consists of exchange $W_{\mathrm{ex}}$, Zeeman $W_{\mathrm{H}}$, and magnetostatic $W_{\mathrm{m}}$ contributions. The exchange and Zeeman terms are not affected by interdot magnetostatic coupling, and hence are the 
dimensional arrays of identical circular dot arrays can be calculated starting from the general expression for the magnetostatic energy density of an in-plane magnetized patterned film [9, 10]. The following expression for the annihilation field can be deduced:

$$
\begin{gathered}
H_{a n}\left(\beta, \delta, R, \varphi_{H}\right)=2 \Gamma\left(\beta, \delta, R, \varphi_{H}\right) M_{s}, \\
\Gamma\left(\beta, \delta, R, \varphi_{H}\right)=\frac{4 \pi}{T_{x} T_{y}} \quad f(\beta k R) \frac{J_{1}^{2}(k R)}{k^{2}} \cos ^{2}\left(\varphi_{\mathbf{k}}-\varphi_{H}\right)-\frac{1}{2} \frac{R_{0}}{R}{ }^{2},
\end{gathered}
$$

where $f(x)=1-(1-\exp (-x)) / x, \beta=L / R$ is the dot aspect-ratio, $J_{1}(\mathrm{x})$ is the Bessel function, and $\varphi_{k}$ and $\varphi_{H}$ are respectively the polar angles for the reciprocal lattice vector $\mathbf{k}$ and applied field $\mathbf{H}$, $T_{\mathrm{x}, \mathrm{y}}=2 R+d_{\mathrm{x}, \mathrm{y}}$ are the dot lattice periods, $R_{0}$ is the exchange length. The function $\Gamma\left(\beta, \delta, R, \varphi_{H}\right)$ defines angular variation of magnetostatically-induced anisotropy. The in-plane initial susceptibility is $\chi(0)=(2 \Gamma)^{-1}$ within the "rigid vortex" model, i.e. it is inversely proportional to $H_{\text {an }}$. The annihilation fields calculated using Eq. 1 (lines) are compared directly to the experimental data (symbols) in inset of Fig. 2. The modeling is in good agreement with the experiments. The same effect of reducing of the vortex nucleation field with decreasing interdot distance was observed and will be reported elsewhere.

The magnetization curve of the rectangular array (B-type) depends on the angle between the external field and the lattice orientation. Fig. 3 shows the angular variation of annihilation field $H_{a n}$ measured for two kinds of rectangular dot arrays with the dot diameter of $0.8 \mu \mathrm{m}$ and the same interdot distance along the [10] lattice direction, but with different distances along the [01] direction, $\left(d_{x}=0.2 \mu \mathrm{m}, d_{y}=1.2 \mu \mathrm{m}\right.$, and $\left.d_{y}=0.4 \mu \mathrm{m}\right)$. Therefore, these two samples can be viewed as a chain and an array of the dots. The variation of annihilation field can be well approximated to the $\sin ^{2}(\varphi)$ function (solid lines), suggesting that both samples have a uniaxial magnetic anisotropy. The easv magnetization axis is narallel to the row of dots with smallest interdot snacing. We found 
that the rule predicted by the "rigid vortex" model $\chi(0) H_{a n} \cup$ const fulfils at changing of the inplane field angle $\varphi_{H}$. The chain, wherein the interdot magnetostatic interaction is more important along one axis than the other, shows stronger coupling effects on the magnetization reversal. The hysteresis loops measured along the hard axis are almost identical to those for the arrays of isolated dots with the same geometry. In the case of rectangular dot arrays, the interdot magnetostatic contributions along different directions are competing, and, as a result, the magnetic anisotropy due to magnetostatic coupling becomes weaker. Finally, square arrays of circular dots with $d_{x}=d_{y}=$ $0.2 \mu \mathrm{m}$ show no in-plane anisotropy, similar to the arrays of magnetostatically isolated dots. We have not found so far any fourfold magnetic anisotropy for the square array as it was reported in Ref. 13 for the FeNi dot arrays with similar parameters. Eq. (1) was obtained in the dipolar approximation. For the considered rectangular arrays near saturation $\left(H=H_{a n}\right)$, the in-dot quadrupole moments are not so important because of the dominant interdot dipolar coupling.

In summary, the magnetization reversal process in the rectangular arrays of circular dots in vortex remanent state is strongly affected by interdot magnetostatic coupling and is collective, when the interdot distance is smaller than the dot radius. The appearance of the dot side surface charges induced by an in-plane external field is responsible for this coupling. The interdot magnetostatic interaction induces an uniaxial magnetic anisotropy, which leads to an decrease of switching fields, an increase in the initial susceptibility, and thus to the vortex instability in the system. The magnetostatically induced anisotropy constant $K_{u}$ is of order $1.7 \times 10^{4} \mathrm{~J} / \mathrm{m} 3$ for the dot arrays with smallest interdot separation. The interdot distance normalized to the dot radius can be used as a key-parameter to determine the strength of the magnetostatic coupling effect.

This work was supported in part by Korea Institute for Advanced Study, RFTF of Japan Society for the Promotion of Science, and the Grant-in-Aid for Scientific Research from the Ministry of Education, Science, and Culture in Japan. Work at ANL was supported by US Department of Enarer REC MAntarin1s Criannas undar nontennt IXI 21 1 NO ENIS 28 


\section{References}

${ }^{1}$ C. A. Ross, Annu. Rev. Mater. Res. 31, 203 (2001).

${ }^{2}$ A. Aharoni, "Introduction to the Theory of Ferromagnetism", Oxford University Press, 2001, 336 pp.

${ }^{3}$ R. P Cowburn, D. K. Koltsov, and A. O. Adeyeye, Phys. Rev. Lett. 83, 1042 (1999).

${ }^{4}$ J. Raabe, R. Pulwey, R. Sattler, T. Schweinbock, J. Zweck, and D. Weiss, J. Appl. Phys. 88, 4437 (2000).

${ }^{5}$ T. Shinjo, T. Okuno, R. Hassdorf, K. Shigeto, and T. Ohno. Science 289, 5481 (2000).

${ }^{6}$ K. Yu. Guslienko, V. Novosad, Y. Otani, H. Shima, and K. Fukamichi, Appl. Phys. Lett. 78, 3848 (2001).

${ }^{7}$ V. Novosad, K. Yu. Guslienko, Y. Otani, H. Shima, K. Fukamichi, N. Kikuchi, O. Kitakami, and Y. Shimada, IEEE Trans. Magn. EMG-37, 2088 (2001).

${ }^{8}$ R. P. Cowburn, A. O. Adeyeye and M. E. Welland, New J. Phys. 1, 16 (1999).

${ }^{9}$ K. Yu. Guslienko, Appl. Phys. Lett. 75, 394 (1999).

${ }^{10}$ E. Y. Tsymbal, Appl. Phys. Lett. 77, 2740 (2000).

${ }^{11}$ K. J. Kirk, J. N. Chapman, S. McVitie, and R. P. Aitchison, J. Appl. Phys. 87, 5105 (2000).

${ }^{12}$ K. Yu. Guslienko, V. Novosad, Y. Otani, H. Shima, and K. Fukamichi, Phys. Rev. B, in press, January 2002.

${ }^{13}$ C. Mathieu, C. Hartmann, M. Bauer, O. Buettner, S. Riedling, B. Roos, S. O. Demokritov, B. Hillebrands, B. Bartenlian, C. Chappert, D. Decanini, F. Rousseaux, Cambril, A. Müller, B. Hoffmann, and U. Hartmann, Appl. Phys. Lett. 70, 2912 (1997). 


\section{Figure captions}

Fig. 1

The scanning electron microscope images and corresponding hysteresis loops of the microfabricated permalloy dot arrays with dot diameter $2 R=0.8 \mu \mathrm{m}$, thickness $L=80 \mathrm{~nm}$ and variable interdot distance $d$.

Fig. 2

The magnetostatically induced anisotropy constant $K_{u} v s$. the normalized interdot distance $\delta=d / R$.

The inset shows scaled annihilation fields $H_{\mathrm{an}} / H_{\mathrm{an}}$ (isol) determined by the experiment (markers) and the calculation (lines) $v s$. the normalized interdot distance $\delta=d / R$. Solid lines are calculated using Eq. (1). The different solid lines corresponds to $\beta=0.40$ (lower curve), 0.27, 0.20 (upper curve). Field is applied along the [10] dot lattice direction.

Fig. 3

Angular variation of the annihilation field $H_{a n}$ measured for two different dot arrays of B-type with dot diameter $0.8 \mu \mathrm{m}$ and interdot distances: $a) d_{x}=0.2 \mu \mathrm{m}, d_{y}=1.2 \mu \mathrm{m}$ and $\left.b\right) d_{x}=0.2 \mu \mathrm{m}, d_{y}=0.4$ $\mu \mathrm{m}$. 


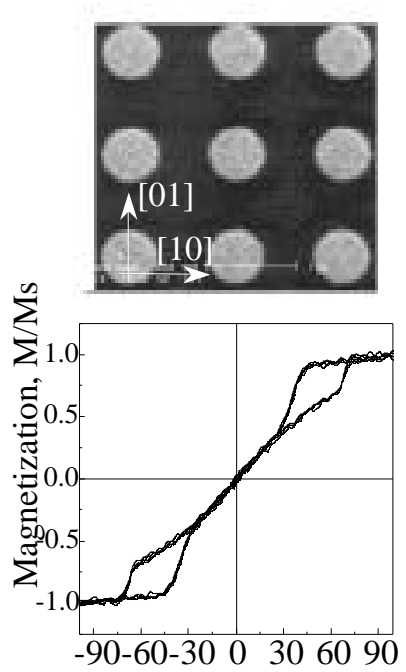

Field, mT
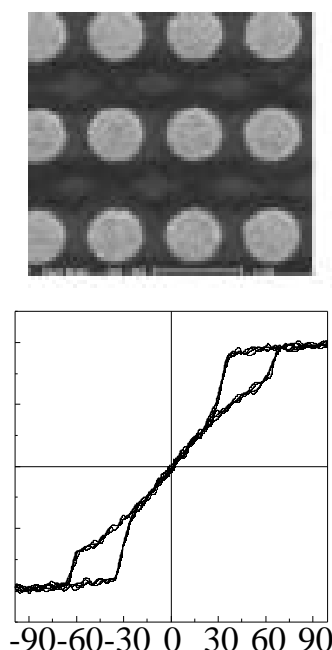

Field, mT
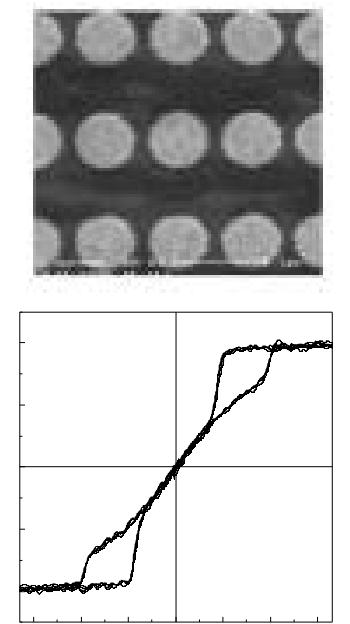

$-90-60-3000306090$ Field, mT
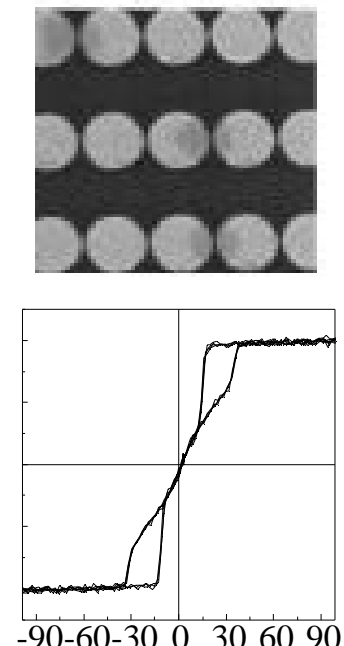

Field, mT

Fig. 1 


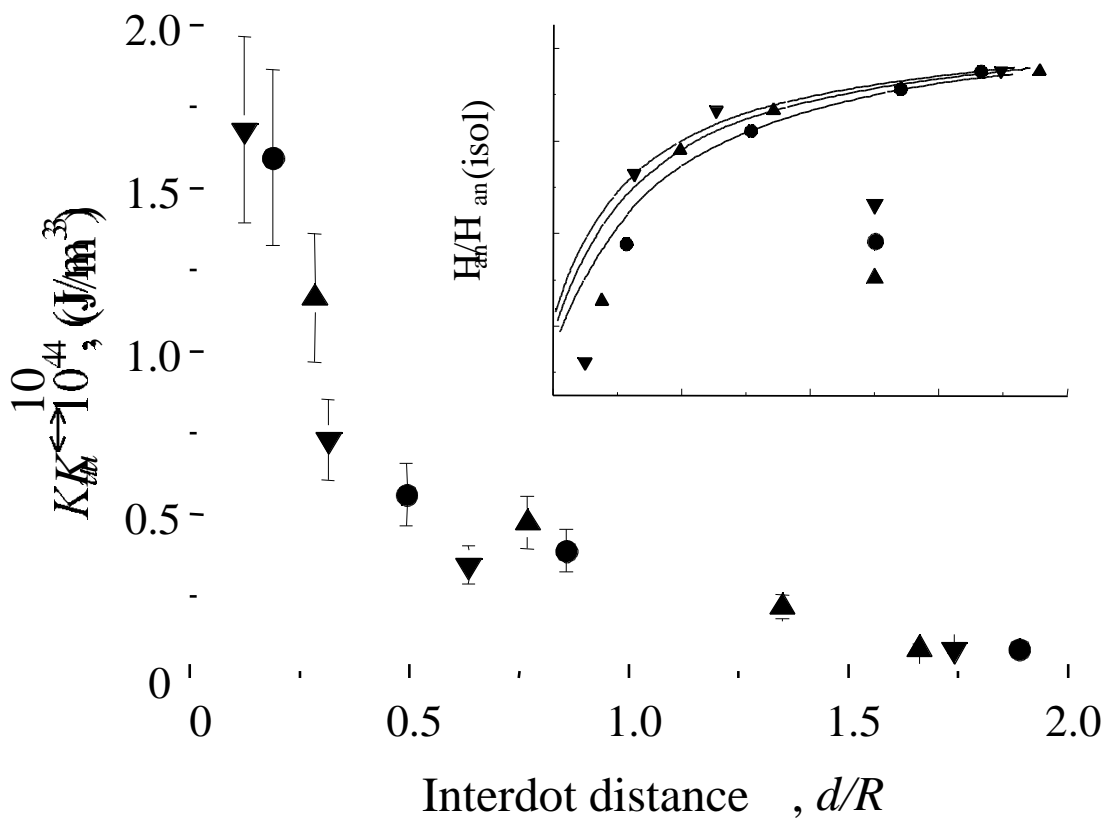


Fig. 2

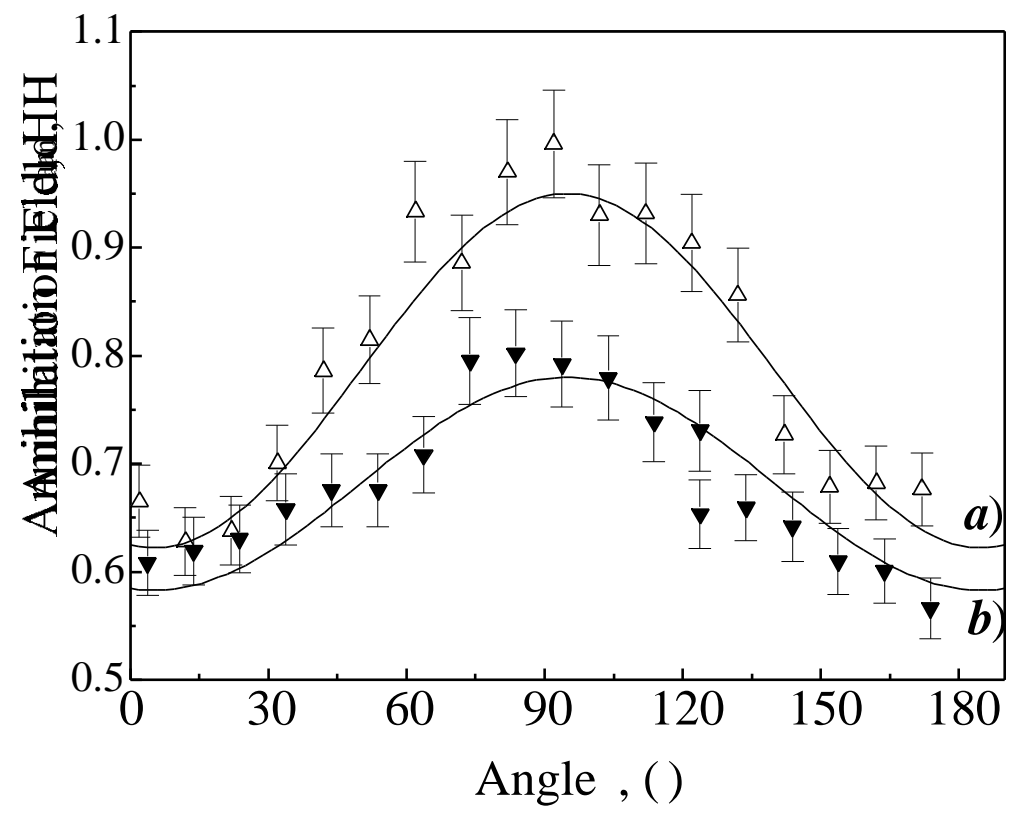


Fig. 3 